\title{
Electrical Impedance Tomography System Based on Active Electrodes
}

\author{
Pascal Olivier Gaggero ${ }^{1,5}$, Andy Adler $^{2}$, Josef \\ Brunner $^{3}$ and Peter Seitz ${ }^{1,4}$ \\ ${ }^{1}$ Centre Suisse d'Electronique et de Microtechnique S.A. (CSEM), \\ Landquart, Switzerland, ${ }^{2}$ Systems and Communication Engineering, \\ Carleton University, Ottawa, Canada, ${ }^{3}$ Swisstom AG, Landquart, \\ Switzerland, ${ }^{4}$ EPFL, Institute of Microengineering, Neuchâtel, \\ Switzerland, ${ }^{5} \mathrm{BFH}$, Institute for human-centered engineering, Bienne, \\ Switzerland \\ E-mail: pascal@gaggero.ch, adler@sce.carleton.ca, jxb@swisstom.com, \\ peter.seitz@epfl.ch
}

\begin{abstract}
EIT can image the distribution of ventilated lung tissue, and is thus a promising technology to help monitor patient breathing to help selection of mechanical ventilation parameters. Two key difficulties in EIT instrumentation make such monitoring difficult: 1) EIT data quality depends on good electrode contact and is sensitive to changes in contact quality, and 2) EIT electrodes are difficult and time consuming to place on patients. This paper presents the design and initial tests of an active electrode based system to address these difficulties. Our active electrode EIT system incorporates an active electrode belt, a central voltage driven current source, central ADCs and DACs, a central FPGA based demodulator and controller. The electrode belt is designed incorporating 32 active electrodes, each of which contains the electronic amplifiers, switches and associated logic. Tests show stable device performance with a convenient ease of use and good imaging ability in volunteer tests.
\end{abstract}

Keywords: electrical impedance tomography, hardware, EIT, active electrode, electrode belt

PACS numbers:

AMS classification scheme numbers:

Submitted to: Physiol. Meas. 


\section{Introduction}

Electrical Impedance Tomography (EIT) produces images of the conductivity within a body from electrical stimulations and measurements at electrodes placed on the body surface. EIT has shown significant promise as a technology to monitor the distribution of air within the lungs of mechanically ventilated patients. Such patients can have highly heterogeneous lungs, and data which EIT can provide are helpful to select lung protective ventilator settings Kunst et al. (2000); Amato et al. (1998). EIT fills a unique niche, by providing tomographic information without a bulky system or ionizing radiation.

Although EIT shows dramatic promise, it is still largely a technology in the research phase. We identify two key factors which impact the reliability and convenience of use of EIT technology. First, EIT is very sensitive to issues associated with the patient interface (i.e. electrodes Soleimani et al. (2006); Lozano et al. (1995) and cables Hong et al. (2007)). The electrode contact to the body tends to have relatively high contact impedance, $Z_{c}$, which also varies with time due to body movement, moisture on the contact surface and electrode replacement. Such high $Z_{c}$ means that all interference sources have a large effect; these include common mode gain error, thermal noise, and EM interference and crosstalk between cables. Second, EIT is often inconvenient to apply since many systems require individual placement of electrodes - which can be especially difficult on the back of heavy or on high-risk patients, which should be minimally disturbed.

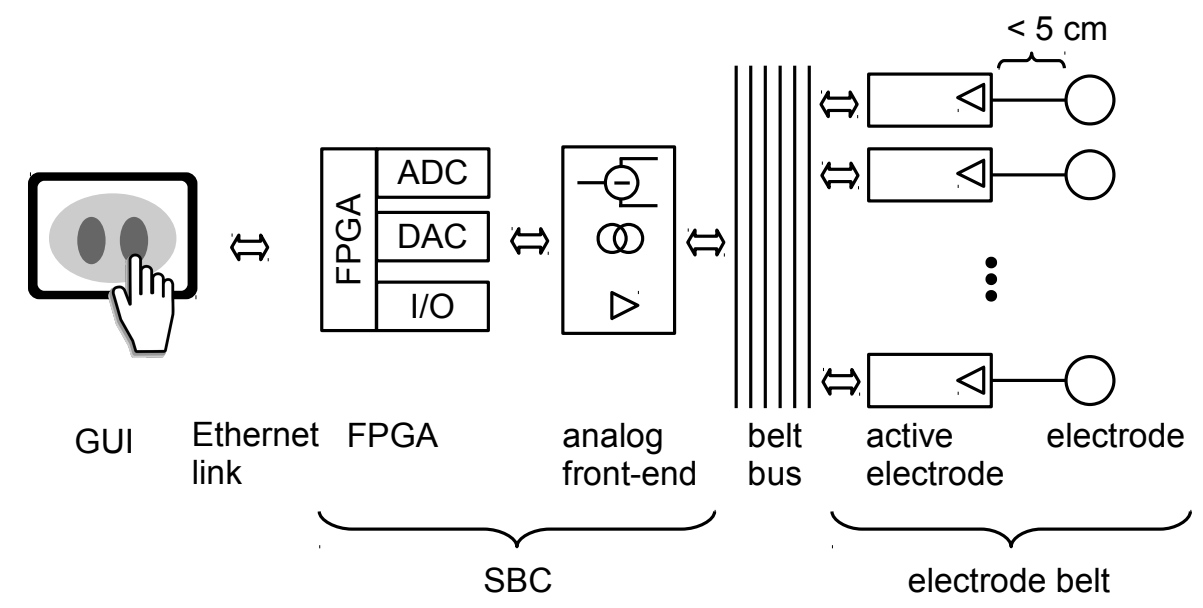

Figure 1. General concept of the designed active electrode based EIT system. The Graphical User Interface (GUI) running on a PC computer is connected to the EIT instrument via an Ethernet connection and communicates using standard TCP/IP protocol. The EIT instrument itself is provided with control and signal processing unit, integrated in the so called Sensor Belt Connector (SBC). The SBC communicates with the active electrode belt using a bus system. Each active electrode is connected to a passive electrode which makes contact with the body, such that the high impedance signal path is less than $5 \mathrm{~cm}$.

This paper describes design and initial testing of an active electrode based EIT system to address the problem mentioned above. The use of active electrodes is motivated by the work of Rigaud et al. (1993), who suggested having active electrodes with embedded current source and voltage buffers, whereas the voltage reference (i.e. signal generator) and the signal demodulation 
are provided at a central location. The analog demodulation required compensation electronics which degrades the impedance and demands calibration procedures. Guardo et al. (1994) described a multiplexed active electrode system with embedded micro-processor to manage the switches. This solution reduces the number of needed cables, compared with Li et al. $(1996 a, b)$, who emphasized the advantage using active electrodes instead of double shielded passive cables. The latter are liable to cause instabilities and are inconveniently thick. Li et al proposed a similar architecture as Guardo et al but without the phase lag compensation. The voltage reading was done only with buffers making use of a dedicated cable (i.e. one dedicated bus line per buffer). In this case the measurement was not multiplexed and the measurement scheme was hardcoded. One of the limitations of the proposed architecture was the high number of cables needed to communicate with the electrodes. Li et al also described the use of electrode gel directly on the electrode surface to "reduce the electrode-interface impedance". The system described in the present article integrates several elements and ideas implemented by the previously cited authors and innovates in the practical implementation of the components of the EIT instrument with the goal of developing a calibration-free, compact and robust commercial grade medical device to serve the intensive care market.

The developed active electrode based EIT system architecture is shown in Fig. 1. This particular active electrode architecture is composed of an active belt, which contains a collection of active electrodes. Each active electrode contains voltage buffers, switches and associated logic. Active electrodes are fabricated into a supported belt which surrounds the thorax, which allows placement of amplifiers and circuit logic as close as possible to the body. In the opinion of the authors the high impedance signal path between the body and the first active element should not exceed $5 \mathrm{~cm}$ in length in order for the system to be labeled "active electrode based". The whole belt is controlled from a central device which integrates control and communication logic, Analog to Digital Converter (ADC), Digital to Analog Converter (DAC), and a unique current source. The logic controller is able to establish an Ethernet connection with a Graphical User Interface (GUI) implemented in a computer software. Using this connection the computer can receive the measured values and send commands to the instrument logic controller.

\section{Hardware design}

\subsection{General consideration}

The key feature of active electrode based EIT instrument architecture is to have a voltage buffer very closely to the body. This offers several advantages compared with the passive electrode architecture: 1) reduces the sensitivity to electromagnetic perturbation, 2) reduces the need for active and / or passive shielding, 3) stabilizes the input impedance of each electrode and 4) reduces, by using in-electrode multiplexing, the number of cables running from the patient to the main electronics.

In practice, an electrode belt will tend to get dirty, for example sweat or blood could enter in contact with the belt structure or the electronics and induce damages. Moreover, there is a hygiene requirement for the belt to be cleaned (or sterilized) between patients. Since washable/sterilizable electronics are still experimental and their reliability not yet demonstrated, we recommend a onetime use belt strategy. Developing a one-time used belt has strong implications on the cost / performance balance, thus we needed to limit the disposable electronics on the belt to the strict minimum. A future implementation of the designed functions in dedicated Application-Specific Integrated Circuits (ASIC) is planned in order to dramatically decrease the cost per active electrode 
compared with a Printed Circuited Board (PCB) based discrete elements solution.

Originally the design concept was to put the electronics as closely as possible to the patient to minimize problems related to the analog transmission of signals on high impedance lines. The most direct way to accomplish that is to place an ADC and a DAC on each electrode and communicate in the digital domain with the rest of the system. Unfortunately, it was not possible to meet the price and size requirements with multiple DACs and ADCs. Therefore we decided to move signal generation and measurement to the central SBC. Having a central and single current source per EIT instrument is probably also an advantage if one desires to monitor the behavior of the current source for electrical safety reasons, for example to prevent more than one of current source injecting current at the same time.

In the following sections we discuss: 1) the design of the active electrode, 2) the design of the central electronics which manages the active electrode network, 3) the special symmetrical current source and 4) the practical implementation of the active electrodes into the thoracic belt.

\subsection{Active electrode}

The concept of active electrodes (see figure 5) is to have active electronic elements as closely as possible to the body. The modes of operation of an active electrode are: 1$)$ injecting a current $\left(\mathrm{SW}_{1}\right.$ closed), 2) sinking a current ( $\mathrm{SW}_{2}$ closed), 3) buffering a voltage and multiplexing the measurement on the analog lines of the bus using switches ( $\mathrm{SW}_{3}$ or $\mathrm{SW}_{4}$ closed) and 4 ) idling while other electrodes are active (all switches open). We use the term "node" for the logic unit which manages active electrode operations. In our design we decided that one node manages one active electrode. The developed prototype makes use of an embedded micro-controller (Atmega328, Atmel, San Jose, California) which contains the state table of the node. At start-up each node takes its reset value and waits for a pulse signal broadcast by the SBC on the sync line (see figure 4). Each time the node measures a pulse, it triggers an interrupt, which fetches the next state in the state table and applies the corresponding configuration to the analog switches. Another possible approach to active electrode control uses a state logic based daisy chain design (see Gaggero (2011)), which does not require an embedded micro-controller. The main focus of this article is to address the most difficult problem of the analog electronics since this presents the biggest challenges and thus we will not enter into the details of the digital management system.

Since the current source is implemented in a central location on the SBC (see section 2.4), the remaining features implemented on active electrodes are essentially signal multiplexing and buffering tasks. The selected architecture uses a fully serial multiplexing strategy, which means that for the current injection there are only two current lines available, one for current source and one for current sink (this feature requires two switches). In order to carry out high-impedance voltage measurements at the electrode, a single operational amplifier configured as a voltage follower is employed. The buffer is followed by two analog switches which multiplex the signal into one of the two possible analog lines. Thus this particular multiplexing architecture only allows for sequential injection and measurement.

One critical factor influencing the noise content of each measurement is the available integration time, thus in a fully serial system the available integration time for each measurement is given by the image rate and the number of measurements to perform. For example, for an image rate of 10 images/s at $32 \cdot 32=1024$ measurements/frame (i.e. for 32 electrodes), there is $\sim 100 \mu$ s for each measurement. The use of a parallel architecture increases the available measurement time to $\sim 3 \mathrm{~ms} /$ measurement, but doing so also increases the necessary hardware and cabling which would 
make the system bulkier and less compact. In this case we chose not to use any parallel measurement strategy to keep the amount of hardware as small as possible.

The use of buffered analog lines versus passive cables has several advantages: 1) offers a better interference and cross-talk immunity (low impedance lines), 2) decreases the input impedance which lowers contact impedance effects, 3) stabilizes the input impedance and 4) removes the requirement for (active) shielded cables. Figure 2 depicts schematically the stray capacitance and contact impedance for the passive electrode architecture and for the active electrode architecture designed in the present work. The input impedance of an active electrode is determined by the stray capacitance of the buffer $(2 \mathrm{pF})$ and other active elements such as the switches for a total of about $15 \mathrm{pF}$. The input impedance of the same electrode but without active elements with $1 \mathrm{~m}$ bus cable and not taking into account multiplexing elements was measured to be at least $60 \mathrm{pF}$ (i.e. measured stray capacitance of a $1.27 \mathrm{~mm}$ pitch flat cable with an adjacent ground line). Routine interference such as touching the cable or changing its geometrical configuration changes the value of the measured stray capacitance between two adjacent lines by up to $10 \mathrm{pF}$.

The main source of measurement variability for passive electrodes is the RC filter formed by the contact impedance $Z_{c}$ and the bus stray capacitance $C_{B}$. Both $\mathrm{R}$ and $\mathrm{C}$ are subject to varying over time. Changes in the contact impedance are generated by 1) variation of the mechanical and geometrical contacts of the electrode with the body, 2) changes in conductivity at the electrodebody interface and 3) most importantly, movement induced contact impedance changes. The latter are induced in a thoracic belt application by the breathing movement of the patient or body posture changes. For a passive EIT system architecture proper active shielding system is required in order to minimize and stabilize the value of $C_{B}$ (Holder (2005)). In contrast, in the active electrode system, the filter formed by $Z_{\mathrm{c}}$ and $C_{B}$ does not exist because by virtue of the voltage buffer the passive electrode is decoupled from the bus line. In this way the signal measured by the electrode is altered by a first filter composed of the contact impedance $Z_{c}$ and two stray capacitances $C_{i n 1}$ and $C_{i n 2}$ related to the operational amplifier stray capacitance and the switches stray capacitance, respectively. Those stray capacitances are stable in time and well defined by the active component characteristics. The only remaining varying element is the contact impedance, which could also be lowered and stabilized using a chemical compound between the electrode and the skin. After the buffer, there is a second filter composed of the on resistance of the analog switch, $R_{o n}$, and the bus stray capacitance $C_{B}$, which can also affect signal integrity. The variations induced by the geometrical changes of the bus line are still present but largely minimized by choosing switches with small $R_{o n}$. Since time difference imaging is not affected by static error induced by stray capacitance and limited operational amplifier open-loop gain, in the following we will only consider contact impedance changes, $\Delta Z_{C}$, which are on the order of $\pm 1 \%$ of the nominal $Z_{C}$ value at the working frequency for our model of the transmission line non-idealities. For example in a thoracic application changes in $Z_{C}$ also occur at the same frequency as the changes of interest inside the body because of the thoracic movement during the ventilation cycle. Additionally there may exist also some long term changes (drift), which invalidate the reference image on the mid/long term (several minutes).

The magnitude of the gain error of the electrode input path for our non-idealities model, assuming a contact impedance change, $\Delta Z_{C}$, in given by:

$$
G_{e r}=1-\frac{1}{\sqrt{1+\left(\omega \Delta Z_{C} C_{\text {stray }}\right)}}
$$




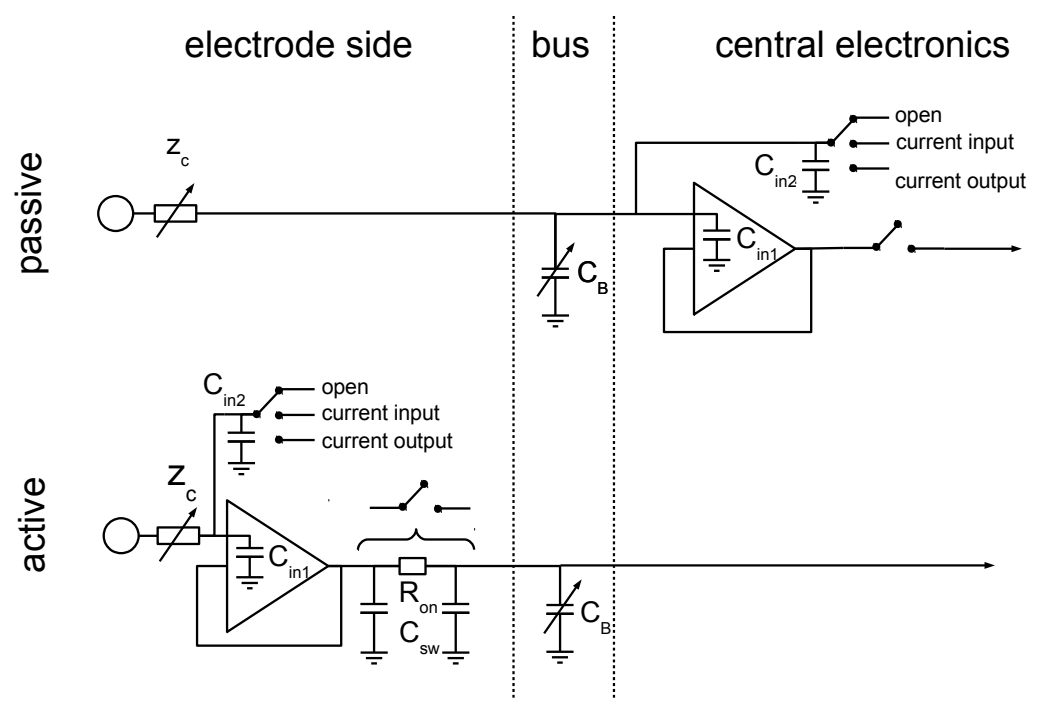

Figure 2. Simplified representation of the stray capacitance and contact impedance for the passive and active electrode architecture.
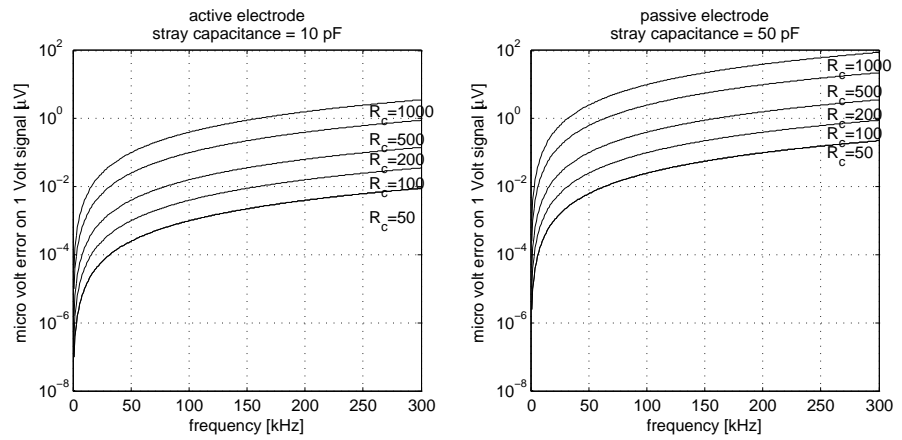

Figure 3. Based on the developed non-idealities model, the resulting voltage errors on a $1 \mathrm{~V}$ signal due to $1 \%$ contact impedance variation for two stray capacitance values of $10 \mathrm{pF}$ and $50 \mathrm{pF}$ corresponding to the model of an active electrode system and passive electrode system, respectively.

It follows that the resulting voltage error assuming an average potential on the body of $U$ is:

$$
\Delta U=U G_{e r}
$$

Considering the case of the thoracic application, and assuming a maximal applicable current of $10 \mathrm{~mA}$ on a $100 \Omega$ impedance, the resulting voltage is $U=1 \mathrm{~V}$. Figure 3 compares the simulation results obtained based on the calculation of the gain error between passive and active electrode architecture using the above described model. These results suggest that active electrodes are less sensitive to contact impedance changes by at least one order of magnitude compared to passively shielded architectures based on non-active electrodes. 


\subsection{Sensor Belt Connector (SBC)}

The sensor belt connector has four main functions: 1) analog signal acquisition, 2) excitation current generation, 3) active electrode node network management and 4) communication and data exchange with the user interface over an Ethernet port. The hardware is composed of two printed circuit boards 1) the Altera Stratix II development kit (EP2S180, Altera, San Jose, California) with integrated ADC (AD9433, Texas Instruments, Dallas, Texas), DAC (DAC904, Texas Instruments, Dallas, Texas) and Ethernet interface, and 2) a custom designed board used as the analog front end.

The analog signal acquisition chain is composed of a differential amplifier (AD8221, Analog Devices, Cambridge, Massachusetts) with a gain $\mathrm{G}_{\mathrm{d}}=10$; it also implements an active High Pass (HP) filter designed to filter out the Direct Current (DC) component of the incoming signal without compromising the settling time excessively. This first amplifier stage is followed by a second whose task is to adjust the signal to the right scale to fit the ADC input range. Right after this last amplification stage the signal is converted into a differential signal, using a fully differential amplifier (THS4502, Texas Instruments) configured to implement an active Low Pass (LP) filter function to avoid aliasing. For the same reason, a second RC low pass filter is implemented as closely as possible to the ADC input pin. The ADC itself is able to acquire the signal with 12 bits resolution at $100 \mathrm{MS} / \mathrm{s}$.

The sinusoidal signal generator is implemented using a FPGA based Numerically Controlled Oscillator (NCO) whose generates a sine signal with a resolution of 14 bits at $100 \mathrm{MS} / \mathrm{s}$, within a frequency range between $10 \mathrm{kHz}$ and $49 \mathrm{MHz}$ with $23 \mathrm{mHz}$ resolution. The amplitude of the signal can also be modified numerically. After leaving the DAC the signal is filtered with a passive band pass filter to suppress DC and high-frequency components. Then the signal is fed into an amplifier whose gain, $G_{c}$, can be chosen to fit the current amplitude needed. The symmetrical current source itself is implemented using a voltage to current converter circuit, which is a slightly modified version of the Howland circuit combined with a voltage source driven by the output voltage of the current source. A symmetrical current source means that the current on the gnd-in line is exactly $180^{\circ}$ dephased compared with the current on the sin-0 line. More detailed information about the current source is given in section 2.4.

The only external digital signal the node network needs to work is a clock signal. After a clock signal is detected each node fetches the next state in the state table and applies the corresponding configuration to the switches. This clock signal is generated by the FPGA logic and buffered before being sent on the bus sync line.

A virtual micro-processor core (NIOSII, Altera) is implemented into the FPGA, such that one can make use of an off the shelf operating system (MicroC/OS-II, Micrium, Weston, Florida) for the TCP/IP implementation. Moreover various features such as task management and prioritization, string manipulation, and many others are already available. Once the SBC is turned on it gets an IP address attributed from a DHCP server or takes the default one. As soon as the user interface implemented with a PC starts a connection, it opens two ports, one for transferring the EIT data and another one for control messages.

\subsection{Central current source}

The voltage signal reference is generated using a DAC and is subsequently buffered to be supplied to a transimpedance circuit, the current source itself. We used a slightly modified Howland current source, which was proposed by Tietze and Schenk (2008). This circuit (see figure 5) acts as a 


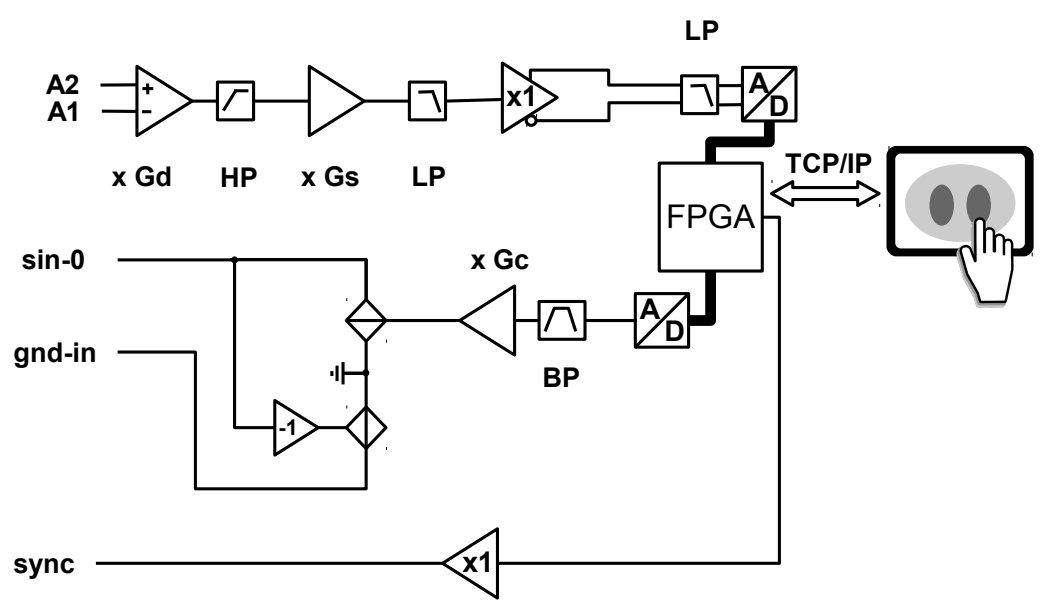

Figure 4. Implementation of the acquisition chain and signal generator on the SBC. The gain of the instrumentation amplifier, $G_{d}$, is set in combination with the gain, $G_{s}$, of the second amplifier stage to best fit the voltage input range of the ADC.

grounded commanded current source using only one operational amplifier and 6 precision resistors for the implementation. It converts a voltage $U_{1}$ into a current $I_{\text {out }}$ according to the relation:

$$
I_{\text {out }}=\frac{U_{1}}{R_{m}}
$$

where $I_{\text {out }}$ is the current injected into the medium, $U_{1}$ is the voltage control signal, $R_{m}$ is the resistor setting the current output for a given value of $U_{1}\left(R_{m}=R_{3}=\mathrm{R}_{6}\right)$. In this calculation the output impedance of the operational-amplifier is assumed to be negligible.

The internal resistance of the current sources is (see Tietze and Schenk (2008)):

$$
R_{\text {int }}=\frac{R_{A} R_{B}\left(R_{A}+R_{B}\right)}{R_{A}^{2}-R_{B}^{2}}
$$

where $R_{A}=R_{2}=R_{5}$ and $R_{B}=R_{4}=R_{1}$. It automatically follows that if $R_{1}=R_{2}=R_{4}=R_{5}$, $R_{\text {int }}$ can be made arbitrarily large. Of course, an obvious limitation appears when taking into account parasitic impedances and the tolerance of individual chip resistors. In the current implementation we use $0.02 \%$ precision resistors. The use of negative impedance converters to further compensate for these effects has been reported in the literature, see for example Holder (2005). The disadvantage, according to these authors, of negative impedance converter appears to be the complexity of the circuit, the individual calibration for each circuit and the long-term stability.

After flowing through the body the current needs to be sinked. Our first approach consisted of simply sinking the current to ground, however tests showed that this approach led to high common mode voltage errors. To solve this issue we implemented a commanded voltage source to "actively" sink the current and balance the injection voltage, which significantly reduced the common mode voltage. This circuit has advantages over classical symmetrical current sources which use a second current source to inject a current with a $180^{\circ}$ phase difference. These systems face the challenge to 


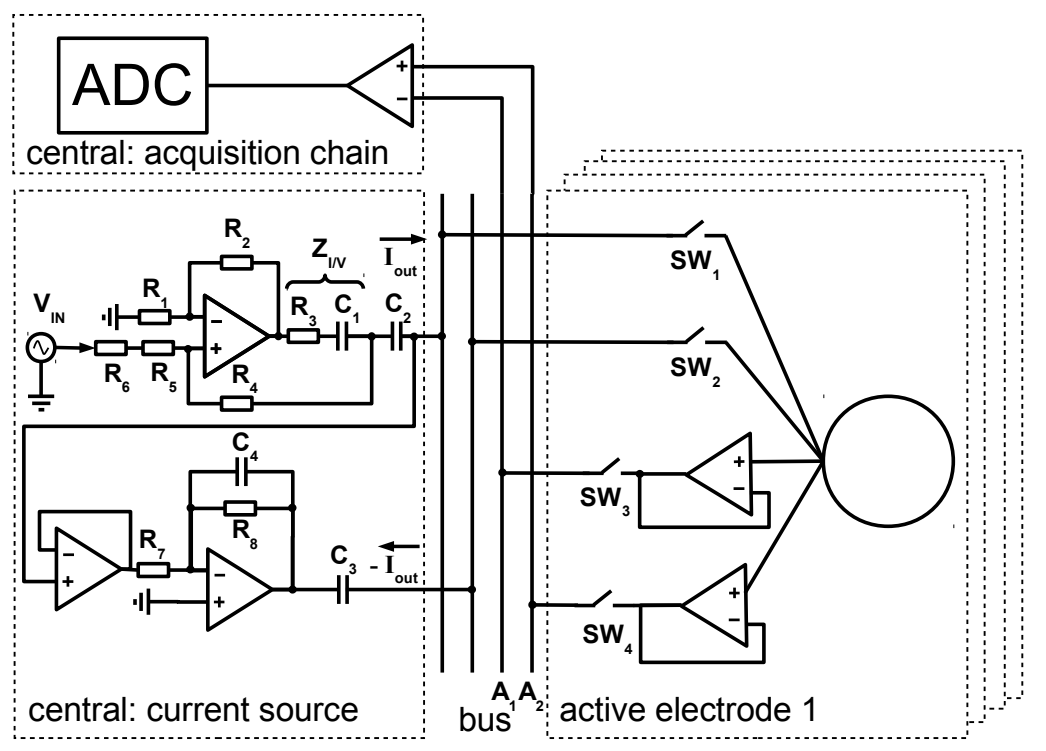

Figure 5. Depicts the overall current injection and voltage measurement scheme. The signals are transmitted using the belt bus and multiplexed using the switches (SW) implemented in each active electrode.

exactly match source and sink current levels, and typically require an additional external ground electrode to absorb current differences, which is not the case for the developed current source.

The circuit used to actively sink the current, and thus to create the symmetrical current is implemented with an operational amplifier configured in unity gain inverter amplifier. Additionally, a capacitor, $C_{4}$, is connected in parallel to $R_{8}$ to dampen high frequency signal components in order to prevent oscillation.

For electrical safety three additional decoupling capacitors, $C_{1,2,3}$, are placed in the current source circuit to avoid the undesired injection of DC into the body. This is advantageous since IEC 60601-1:2005 safety limits are much lower for DC that for AC (see IEC (2005)). Moreover when injecting DC into a medium using metallic electrodes, the latter undergo undesired redox chemical reactions which ultimately create a drift in the signal.

\subsection{Electrode belt}

In this work, we identify the individual placement of several discrete electrodes as one of the obstacles preventing EIT to be widely used in clinical settings. Thus we propose gathering all active electrodes on a belt like structure. The constructed belt is made of a bio-compatible fabric, also used for wound bandages. The active electrodes are electrically linked together using short Flexible Flat Cable (FFC) pieces, each with 16 wires. The construction ensures a certain stretchability in the length of the whole belt structure, such that the belt induced mechanical constraints on the thorax are minimized. For this prototype we use a gold plated copper electrode directly soldered on the top of the active electrode PCBs as shown in figure 6 a). 


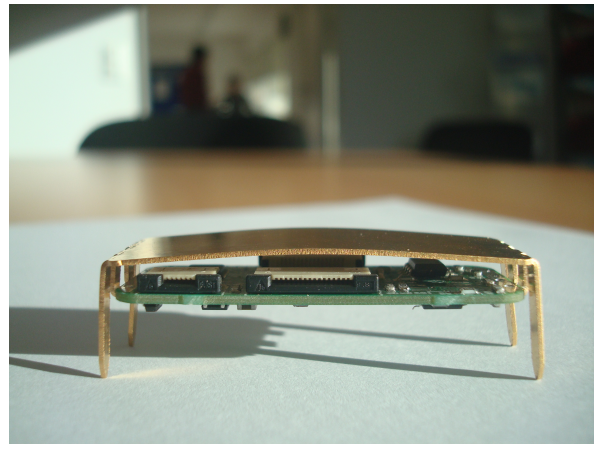

(a)

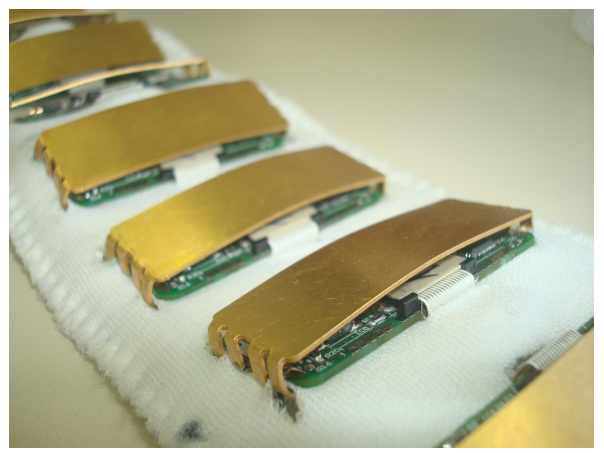

(c)

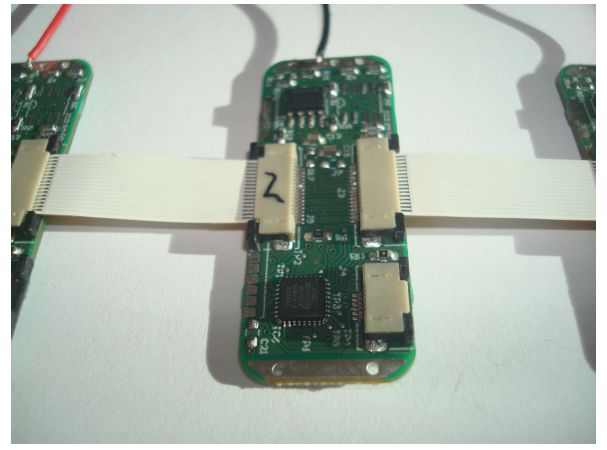

(b)

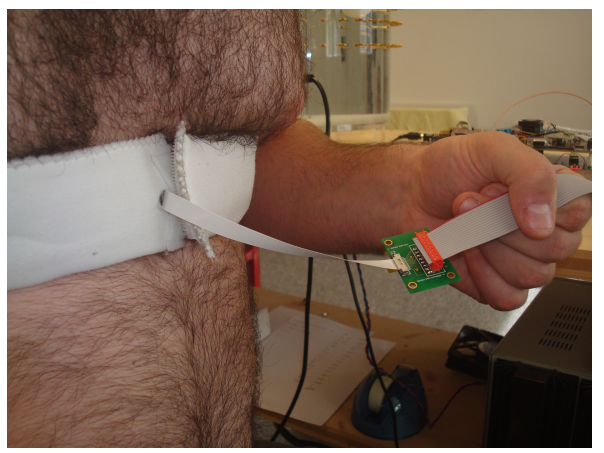

(d)

Figure 6. Illustration of developed thoracic-EIT system. a) active electrode PCB with goldplated copper electrode, b) active electrodes with micro-processor, switches and buffer are connected in a belt-like structure using FFC cables, c) belt-like structure can be incorporated within bio-compatible fabric and d) active electrode belt worn by male volunteer.

\section{Tests}

\subsection{Current source internal impedance measurement}

The aim of this test is to determine the internal impedance of the symmetrical current source. The test scheme is shown in figure 7 . The load resistor $R_{L}$ is varied from $0 \Omega$ to $1150 \Omega$, then for each selected load resistor value the current $I_{1}$ and the voltage $V$ are recorded. Applying basic circuit theory to the circuit figure 7 , one can calculate an expression for the internal impedance as follow:

$$
V=I_{2} Z
$$

where $I_{2}=I-I_{1}$; thus one can write:

$$
V=\left(I-I_{1}\right) Z
$$




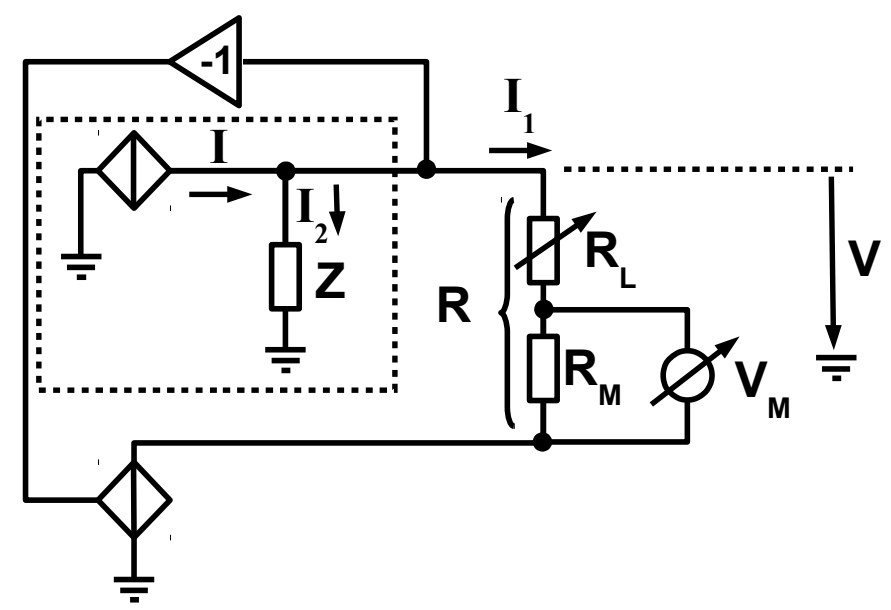

Figure 7. Electronic circuit used to measure the internal impedance of the designed current source.

which after algebraic manipulation becomes:

$$
-\frac{V}{Z}+I=I_{1}
$$

where $V$ is the voltage drop as indicated in figure $7[\mathrm{~V}], R_{M}$ is the fixed measurement resistor of $150 \Omega$ used to determine the current $I_{1}, R_{L}$ is the variable load resistor $[\Omega], I$ is the current produced by the ideal current source $[\mathrm{A}], I_{1}$ is the current flowing through the resistor $R[\mathrm{~A}], I_{2}$ is the current flowing through the internal impedance $Z[\mathrm{~A}], Z$ is the internal impedance of the current source $[\Omega]$.

Equation 6 can be identified as a straight line equation of the type $a x+b=y$ where the slope is given by $a=-\frac{1}{Z}$. Therefore, using the experimental result presented in figure 8 , it is possible to extrapolate the current source's internal impedance from the slope of the obtained straight line. The effect appears not to be linear, thus the idea is to fit the data with two straight lines, one for load resistances under $550 \Omega$ (first 3 data points) and one for load resistances above $550 \Omega$. Then from each line equation one gets the slope value that corresponds to the internal impedance of the source. Table 1 presents the estimated value of the internal impedance calculated from the data displayed in figure 8. From these internal impedance data, one can conclude that as far as the internal impedance of the current source is concerned the most favorable working frequency in this case is $50 \mathrm{kHz}$. This is consistent with the expected behavior that the lower the frequency the smaller is the effect of the stray capacitance. Thus the benefit of lower excitation frequency is an improved internal impedance performance. However increasing the excitation frequency allows injecting more current into the body (i.e. up to $10 \mathrm{~mA}$ above $100 \mathrm{kHz}$ according to IEC 60601-1:2005 (see IEC (2005))), and it is also easier for the current to flow through the body due to lower contact impedance. Moreover working with low excitation frequency also restrains the achievable frame rate, because at least one complete sine period is needed to correctly demodulate the signal amplitude. In the case of a fully serial 32-electrode EIT system working at $10 \mathrm{kHz}$ the maximal frame rate would be 10 images per second, which is already fairly slow. Thus a tradeoff between current source internal impedance, excitation frequency, contact impedance, frame rate and number of electrodes should be found. For 


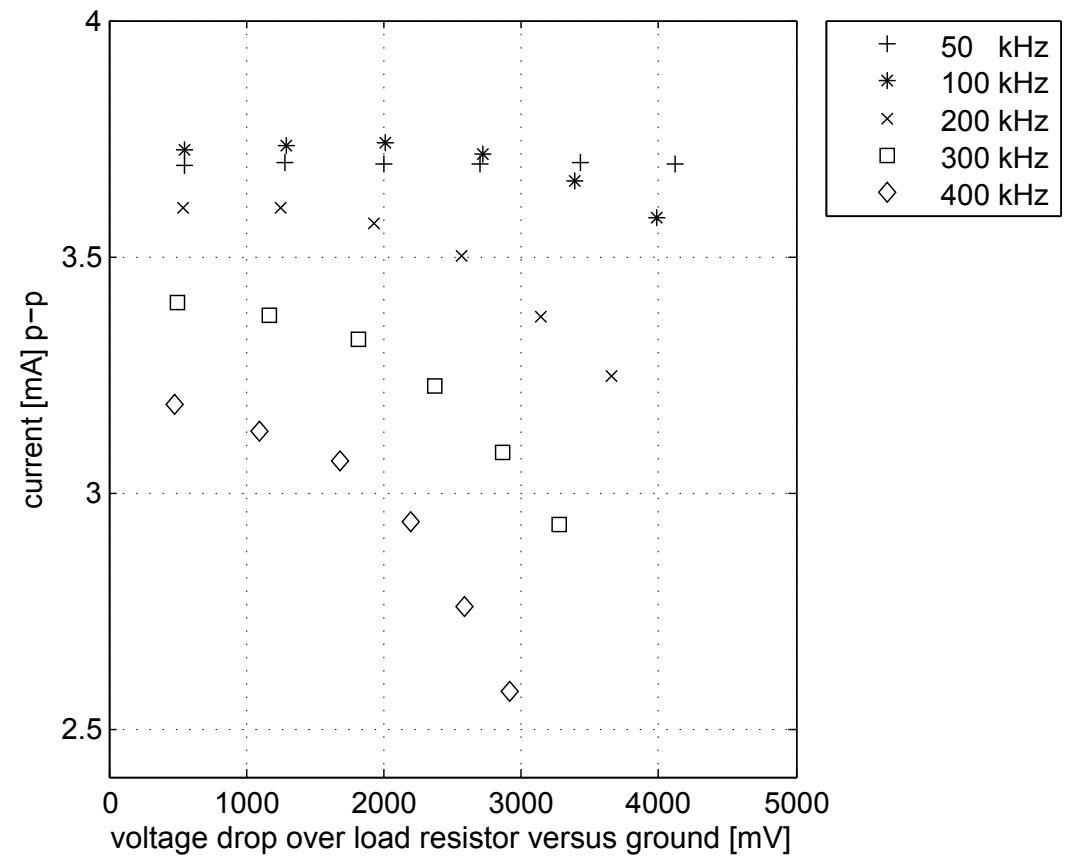

Figure 8. The output current versus output voltage diagram of the current source for various frequencies and load conditions with a fixed current setting.

the developed system a qualitative data analysis on figure 8 suggests that an appropriate frequency range is between 50 and $200 \mathrm{kHz}$. This is similar to specifications published by other EIT system manufacturer: Dräger Medical AG Pulmovista 500 (see Minner (2010); Dräger (2011)) EIT system current excitation frequency range is 80 to $130 \mathrm{kHz}$ and the GOE MF II (see Hahn et al. (2002)) works at $50 \mathrm{kHz}$.

Table 1. The internal impedance measurement corresponding to the data depicted in figure 8 . The data corresponding to the $50 \mathrm{kHz}$ measurement are approximately aligned on a horizontal line whose slope is near zero. The numerical results around zero are very sensitive to measurement error, which is why the numerical values for the $50 \mathrm{kHz}$ line are given in parentheses. The negative internal impedance for the $100 \mathrm{kHz}$ measurement is not a mistake and it can be consistently reproduced.

\begin{tabular}{ccc}
\hline frequency $[\mathrm{kHz}]$ & $\begin{array}{c}\text { internal impedance for } \\
R \leq 550 \Omega[\mathrm{k} \Omega]\end{array}$ & $\begin{array}{c}\text { internal impedance for } \\
R>550 \Omega[\mathrm{k} \Omega]\end{array}$ \\
\hline \hline 50 & $(-1058)$ & $(-2000)$ \\
\hline 100 & -116 & 9 \\
\hline 200 & 44 & 4 \\
\hline 300 & 17 & 3 \\
\hline 400 & 10 & 2 \\
\hline
\end{tabular}




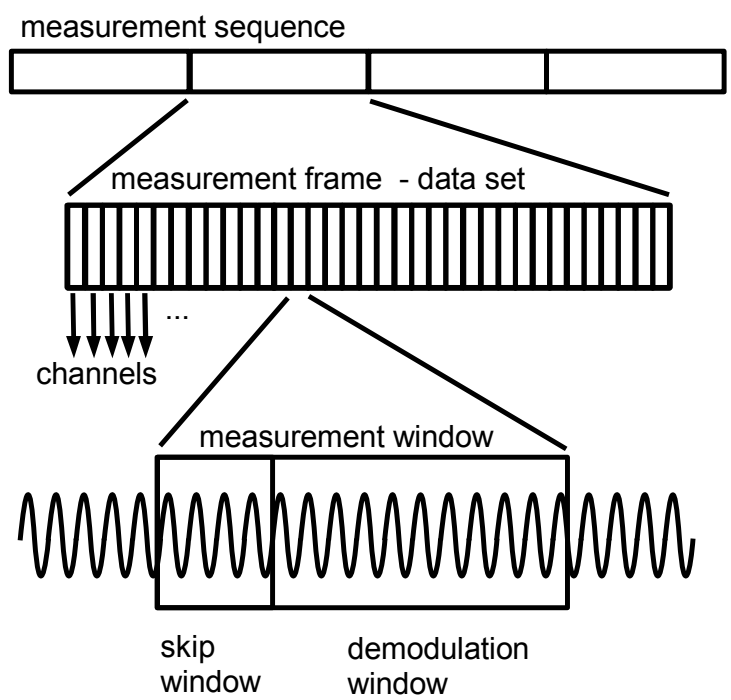

Figure 9. Illustration of the EIT signal sequence obtained with a fully serial system. The signal finally used to be demodulated is selected by adjusting the skip and demodulation windows length.

\subsection{Multiplexing noise}

The developed EIT system is fully serial, in other words, only one single measurement by virtue of a pair of active electrodes is performed at the same time. The instrumentation amplifier (AD8221, Analog Devices), which determines the analog difference between both analog lines, needs a certain time before the signal at its output meets the stability requirements and is ready for use. According to the data sheet a $10 \mathrm{~V}$ step is stabilized after $13 \mu \mathrm{s}$ within $0.001 \%$ of precision. Since during the transition states between two measurements the output of the AD8221 can reach its power supply rails $( \pm 5 \mathrm{~V})$, we require the system to wait 15 to $30 \mu$ s before demodulating the signal. The exact value and state of the input and output of the instrumentation amplifier during the transition phase is not controlled; this is why the settling time varies so much from one measurement to another.

Clearly, there are some techniques that can be used in future systems to ensure cleaner transitions. For instance, both analog lines could be short-circuited during transitions; it should provide the system with a much more stable settling time. Indeed for differential imaging, it is only important to stabilize the transition time to get a better system accuracy.

The strategy used in the developed prototype to mitigate the transition time dependencies of the measurements is to wait for a constant amount of time at the beginning of each measurement window. This waiting time allows the signal to stabilize. The digital IQ-demodulator can skip integer numbers of signal periods before starting to demodulate the incoming signal (see figure 9: skip window). By varying the length of the skip window, one can measure the best trade-off between the waiting time and the measurement time. The measurement window length is given by the number of measurements per frame (image) and the image rate. From signal processing theory, it is known that one should maximize the integration time of the signal in the demodulator to reach the best signal to noise ratio. But one also knows that if one does not wait until the signal is 


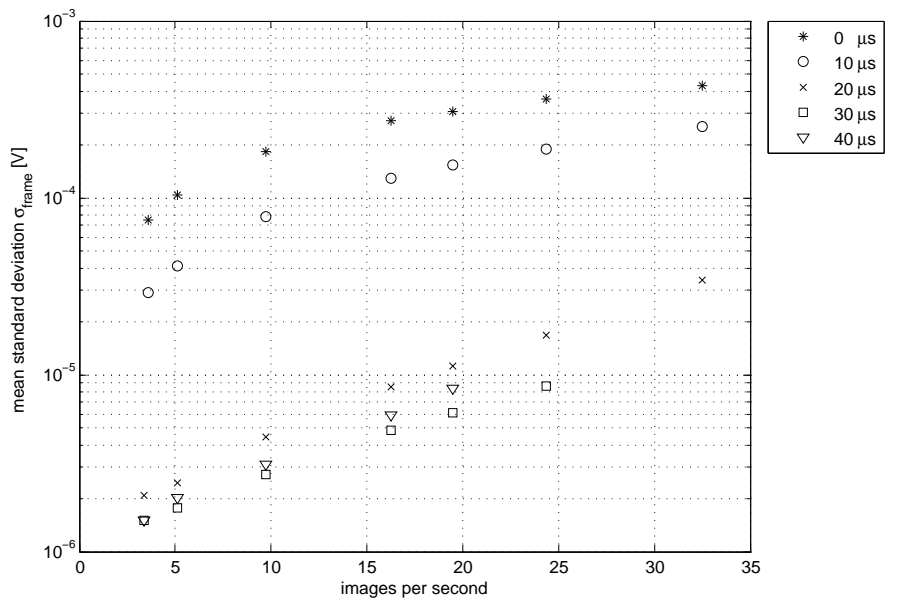

Figure 10. The effect of the skipped time (in $\mu$ s) on the frame noise has been studied in function of the frame rate. The working frequency for these measurements was $100 \mathrm{kHz}$.

stabilized before demodulation begins, one will introduce a large amount of multiplexing noise into the demodulated values. In order to measure the best trade-off between skip window length and demodulation window length for the developed EIT system, one can design a simple experimental set-up composed of the 32 active electrodes system placed around a saline phantom (see Mamatjan et al. (2011)) without any object inside. The data are recorded for various image rates and waiting times, then for each parameter combination, the noise content is assessed and reported on a graph. Since each channel has potentially its own noise value, a way to assess the noise of one data frame is to sum the power of the noise and normalize by the number of measurements (i.e. number of channels):

$$
\sigma_{\text {frame }}=\sqrt{\frac{1}{N_{c h}} \sum_{i=1}^{N_{c h}} \sigma_{i}^{2}}
$$

where $N_{c h}$ is the number of channels, $\sigma_{i}$ is the noise of the i-th channel, $\sigma_{\text {frame }}$ is the mean noise for the whole measurement frame (frame noise).

In figure 10, one can observe that for a waiting time of $30 \mu$ s the frame noise is minimal among all tested frame rates. This value is a compromise between the integration time (demodulation window) and the available measurement window length. In this particular case waiting $40 \mu$ s before demodulating the signal exhibits worse noise performance than the "skip $30 \mu \mathrm{s}$ " strategy. Waiting only $20 \mu \mathrm{s}$ is suboptimal in terms of noise performance, but enables to reach much higher frame rates (i.e. at least one complete sine period is necessary to demodulate the signal correctly).

\subsection{A first qualitative test on a human volunteer}

The aim of the present section is to qualitatively demonstrate the feasibility of thoracic imaging using the presently described hardware and the ability of the resulting EIT data to deliver physiological relevant information. For this purpose we conducted an experiment on a healthy 
male volunteer in sitting posture. Before the experiment the EIT instrument is warmed-up during at least 10 minutes in order for the electronics to stabilize in temperature. Then prior to the test the skin surface is prepared using a liquid, to lower and stabilized the contact impedance between the skin and the electrode. Then the belt is placed around the thorax in a transverse plane crossing the $5^{\text {th }}$ ribs and the sternum. The data acquisition system is set to work with $2 \mathrm{~mA}$ peak to peak at $100 \mathrm{kHz}$ with a skip window length of $30 \mu \mathrm{s}$. As soon as the system is running the operator sets the reference image at the end of the exhalation.

In the example depicted in figure 11 the volunteer is asked to slowly take a deep breath. At the beginning (1) of the respiratory cycle the diaphragm is in a relaxed state. For the air inhalation to be initiated, the diaphragm contracts which reduces the pressure inside the lungs. This creates a pressure gradient that causes air to flow (2) in the lungs through the trachea. This process continues until the volunteer stops taking air in (4) and begins exhaling the air out of his lungs (6). This inhalation and exhalation cycle can conveniently be visualized on the EIT composite signal, which is the mean of used differential voltage measurements given by:

$$
\overline{V_{c}}=\frac{1}{N} \sum_{i=1}^{N} V_{i}
$$

where $N$ is the number of voltage measurements used to form the image, $V_{i}$ are the individual voltage measurements.

\section{Discussion}

EIT has shown a significant promise of medical applications, mainly, but not only, for ventilation monitoring. One key drawback has been limits to the robustness and ease of use of EIT systems, which encouraged us to develop a new instrument based on the active electrode technology. This paper describes our EIT design, an active electrode system system using a 32-electrode biocompatible fabric belt, linked to a small-sized central electronics box, that sends the data to a remote host device such as a computer or a ventilator.

In this work we successfully demonstrated the use of active electrodes for human thoracic EIT applications. We highlight the fact that designing an EIT system is based on compromising several parameters such as: the amount of necessary hardware, the excitation frequency, the contact impedance, the frame rate and the number of electrodes. We experimentally found that considering a fully serial 32 active electrode system with a working frequency between $80-200 \mathrm{kHz}$ and a frame rate between 10 and 30 images per second seems reasonable settings. Next steps for the hardware development are the further miniaturization of the electronics on each PCB and better integration of the belt bus. The latter could integrate the bus, the passive electrode and soldering pads for the PCBs all together on the same support. Overall this work was motivated by the authors' belief that a broad clinic acceptance of EIT is closely linked to the system robustness and ease of handling, thus using active electrodes integrated in a unique belt structure constitutes an important step towards this goal. 

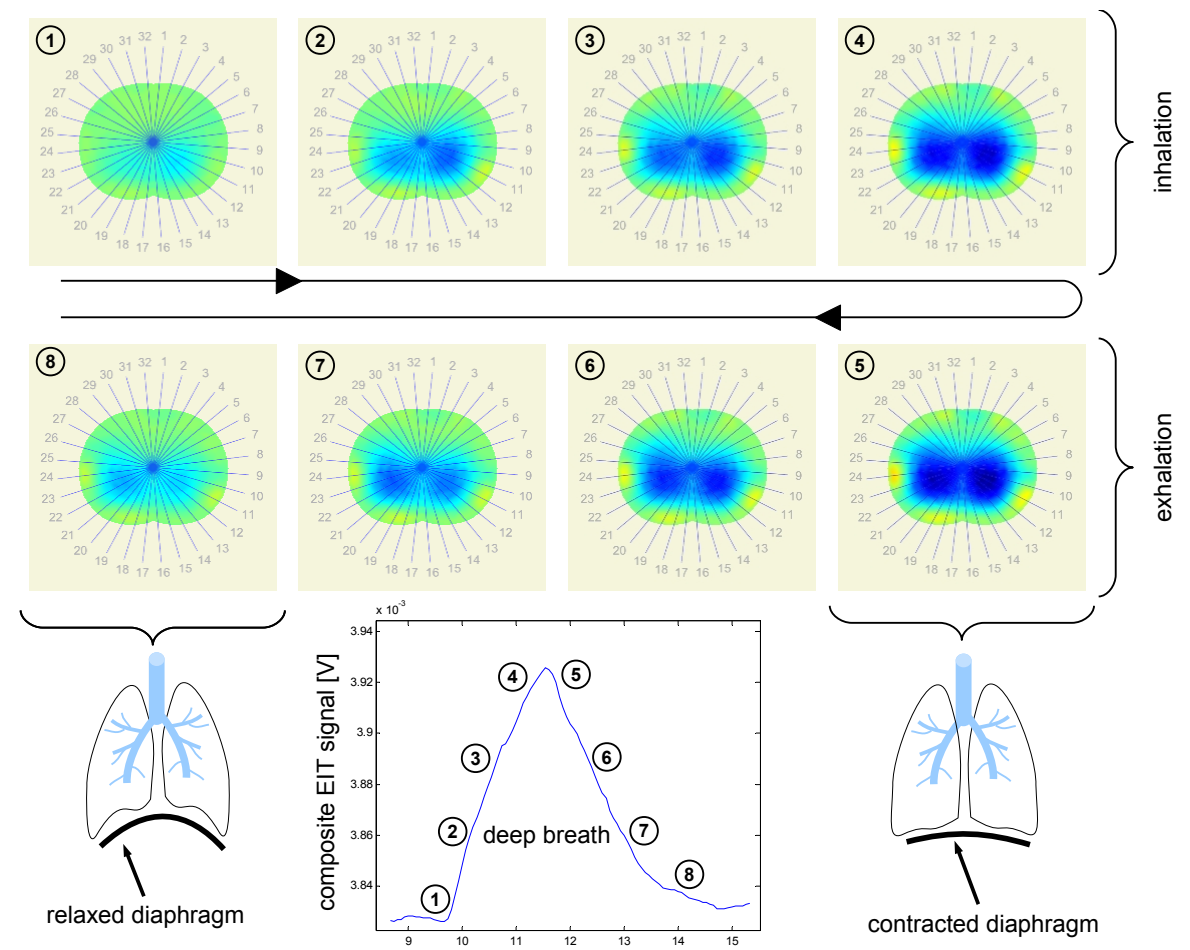

Figure 11. EIT tomographic images of the thorax region during one inhalation-exhalation cycle. The plot depicts the composite EIT signal (see equation 8) of a volunteer taking a deep breath.

\section{References}

Amato M B, Barbas C S, Medeiros D M, Magaldi R B, Schettino G P, Lorenzi-Filho G, Kairalla R A, Deheinzelin D, Munoz C, Oliveira R and et al. 1998 Effect of a protective-ventilation strategy on mortality in the acute respiratory distress syndrome. The New England Journal of Medicine 338(6), 347-354.

Dräger 2011 Dräger Pulmo Vista 500 Data Sheet.

Gaggero P O 2011 Miniaturization and distinguishability limits of electrical impedence tomography for biomedical application $\mathrm{PhD}$ thesis Université de Neuchâtel.

Guardo R, Boulay C, Provost B, Bohsina D and Mallette S 1994 Micro-controller based active electrodes for impedance tomography Engineering in Medicine and Biology Society 1, $545-546$.

Hahn G, Dudykevych T, Frerichs I, Thiel F and Hellige G 2002 A high performance electrical impedance tomography (eit) system for clinical evaluation studies and space application Proc Conf 2nd European Medical and Biol Eng pp. 110-111.

Holder D 2005 Electrical Impedance Tomography 1nd, edn Institute of Physics Bristol, UK. 
Hong S, Yan W, Shu Z and Chaoshi R 2007 Integrated data collection in electrical impedance tomography 13th International Conference on Electrical Bioimpedance and the 8th Conference on Electrical Impedance Tomography 17, 348-351.

IEC 2005 Medical electrical equipment Part 1: General requirements for basic safety and essential performance, IEC 60601-1:2005.

Kunst P W, Vazquez De Anda G, Böhm S H, Faes T J, Lachmann B, Postmus P E and De Vries P M 2000 Monitoring of recruitment and derecruitment by electrical impedance tomography in a model of acute lung injury. Critical Care Medicine 28(12), 3891-3895.

Li J H, Joppek C and Faust U 1996a Fast eit data acquisition system with active electrodes and its application to cardiac imaging. Physiological Measurement 17 Suppl 4A, A25-A32.

Li J H, Joppek C and Faust U $1996 b$ In vivo eit electrode system with 32 interlaced active electrodes. Medical $\& 3$ Biological Engineering 83 Computing 34(3), 253-256.

Lozano A, Rosell J and Pallas-Areny R 1995 Errors in prolonged electrical impedance measurements due to electrode repositioning and postural changes. Physiological Measurement 16(2), 121-130.

Mamatjan Y, Boehm S, Gaggero P O and Adler A 2011 Evaluation of eit system performance. Physiological Measurement 32(7), 851-865.

Minner J 2010 Die Elektroimpedanztomographie als Verfahren zur Lageevaluation des Doppellumentubus PhD thesis Albert-Ludwigs-Universität Freiburg im Breisgau.

Rigaud B, Shi Y, Chauveau N and Morucci J 1993 Experimental acquisition system for impedance tomography with active electrode approach. Medical \&s Biological Engineering 83 Computing 31, 593-599.

Soleimani M, Gomez-Laberge C and Adler A 2006 Imaging of conductivity changes and electrode movement in eit Physiological Measurement 27(5), S103-S113.

Tietze U and Schenk C 2008 Electronic Circuits 2nd, edn Springer Berlin, DE. 